\title{
The Research of Weak Signal with Unknown Frequency Detection Based on the Compound System
}

\author{
Hongyu Zhang ${ }^{1}$, Yihe Wang ${ }^{2}$, Xingyu Liu ${ }^{1}$, Gang Zhao ${ }^{2}$ and Meiyan Shan ${ }^{2}$ \\ 1 State Grid Liaoning Electric Power Supply Co. Ltd., Liaoning Province, China \\ 2 State Grid Shenyang Electric Power Supply Co. Ltd., Liaoning Province, China
}

\begin{abstract}
Aiming at the difficult problem of weak signal detection, a weak signal detection method based on the compound system for weak signal with unknown frequency is proposed, which implements the detection of weak signal submerged in strong noise and provides for the basic theory and technical support for the protection of industrial processes to efficient and stable operation, using the sensitivity of the state transitions of the phase trajectories of the system (from the chaotic state to the large-scale periodic state) to the weak signal input, combining with the filter capability of the correlation method.
\end{abstract}

Keywords: weak signal detection, compound system; filter capability; stable operation

\section{Introduction}

In weak signal detection process, the signal input to the detection system is often accompanied by the noise $[1,2]$. The word weak in weak signal detection not only refers that the amplitude of signal to be measured is low, and means that the ratio of the measured signal amplitude and noise power is low. That is to say, the purpose of the weak signal detection is to detect weak signal submerged in strong noise. Therefore, the noise is unfavorable factors in the processing of weak signal detection. How to suppress noise to improve effectively the SNR threshold is a difficult problem to be solved in the field of weak signal detection.

In view of the weak signal detection technology, the foreign develops rapidly. In 1942 the establishment of Wiener filtering theory [3-5] greatly promoted the development of the weak signal detection theory in the world, and at the same time, domestic personnel engaged in related research is less. From then on, all kinds of new method of weak signal detection theory emerge in endlessly and a variety of excellent performance testing equipment have been introduced which greatly improves the detection accuracy of weak signal. The detection of weak fault signal using chaotic data sequence analysis and fault diagnosis technology has been up in recent years, and becomes a new and effective method [6-10]. Therefore, the combination of the chaos theory and weak signal detection is of epoch-making significance, it opens up a new application field of chaos theory, actually provides a new theoretical foundation and support for signal detection technology in the application of engineering.

With the development of weak signal detection, the types of weak signal increases, the requirements for detection accuracy are also getting higher and higher. The method of using a single weak signal detection hasn't gradually meet the detection needs of complex industrial process. A method mentioned in the literature [11] of using the sensibility of Duffing system to weak periodical signal which has small reference signal frequency offset realizes the goal of detecting the signal with unknown frequency. However, since the result of detection is strongly influenced by noise and signal to noise ratio threshold of detection is rather high, the method fails to guarantee higher detection 
accuracy. Therefore, it has become a new hotspot to adopt a combination of a variety of detection methods and gives full play to the advantages of detection means.

This paper presents a compound system for unknown frequency weak signal detection that combines the detection method of double coupling Duffing oscillator and crosscorrelation method. This method can fully play their respective advantages and extract periodic signal under test from strong noise that greatly reduces the SNR threshold of the signal.

\section{Weak Signal Detection with Unknown Frequency based on the Double Coupling Doffing Oscillator}

1) The characteristics of double coupling Duffing system

The specific form of equation for the single Duffing is defined as

$$
\ddot{x}(t)+k \dot{x}(t)-x(t)+x^{3}(t)=F \cos (\omega t)
$$

Where, $x(t)$ is variable of chaotic system, $k$ is damping ratio, $t$ is time variable, $F \cos (\omega t)$ is periodic driving force, where $F$ is periodic driving force amplitude and $\omega$ is the angular frequency of the driving cycle. $-x(t)+x^{3}(t)$ is the nonlinear restoring force. In the case of k stationary, the system state changes regularly with the amplitude of driving force $F \cos (\omega t)$.

Two coupled Duffing system, a kind of improved Duffing system, is coupled up with the two single Duffing system in order to eliminate some noise through coupling. Its concrete form is defined as

$$
\left\{\begin{aligned}
\ddot{x}(t) & +k \dot{x}(t)-x(t)+x^{3}(t)+d(y(t)-x(t)) \\
& =F \cos (\omega t) \\
\ddot{y}(t) & +k \dot{y}(t)-y(t)+y^{3}(t)+d(x(t)-y(t)) \\
& =F \cos (\omega t)+Y(t)
\end{aligned}\right.
$$

Where, $\mathrm{x}$ is the variable of the first Duffing system, $\mathrm{y}$ is the variable of the second Duffing system, $d$ is coupling coefficient (in this paper, it is $\mathrm{d}=2$ ), when it is $d=0$ coupling of the two systems disappears, dynamic behavior of the system and the single Duffing oscillator system behavior are identical. When it is $d \neq 0$ variables $\mathrm{x}, \mathrm{y}$ of the two system rapidly tend to synchronization on the effect of coupling. $Y(t)$ is external input signal of system including the measured signal $A s(t)$ and the noise signal $\eta(t), A$ is the amplitude of the measured periodic signal and the measured signal frequency has nothing to do with the driving frequency. Under the condition of fixed damping ratio, phase trajectory of two coupled Duffing system $F \cos (\omega t)$ changes with the amplitude of driving force.

\section{2) The detection process}

A method for detecting weak signal with unknown frequency based on double coupling Duffing oscillator consists of two detection links: frequency detection link and amplitude detection link. The detection process is just like Fig.1, the measured signal containing noisy is input to double coupling Duffing system, then it can detect the frequency of the measured signal through piecewise measuring frequency detection method. In the process of the amplitude detection, firstly the reference frequency of the driving signal is measured as the measured signal. Then the measured signal with noisy is input to double coupling Duffing system, detecting amplitude of the measured signal. 


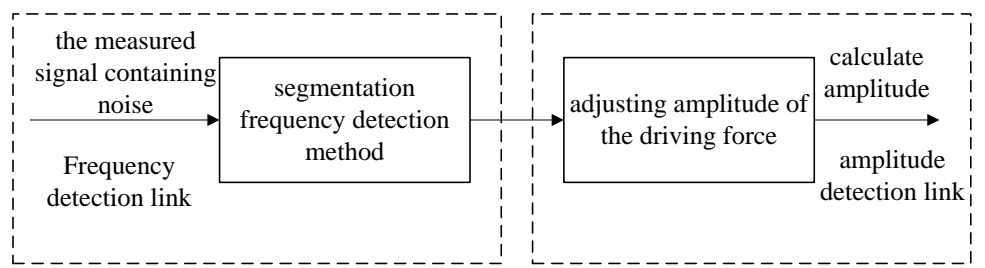

Figure 1. The Detection Process of Detecting Weak Signal Method with Unknown Frequency based on Double Coupling Duffing Oscillator

\section{Weak Signal Detection Based On Cross-Correlation Method}

In the method of weak signal detection, time domain method is the first developed detection method and related method is one of the most commonly used traditional time-domain detection method. The basic idea of weak signal detection based on cross-correlation method is that, in the situation of the measured signal with known frequency, the measured signal mixed with noise and reference signal with the same frequency is made cross-correlation arithmetic[12-17], finally the magnitude of the measured signal is determined according to the output of amplitude of correlation function. Weak signal detection based on the autocorrelation method is to deal with the measured signal and itself, regarded as a special case of cross-correlation method.

The input signal mixed with noise is defined as

$$
\mathrm{F}_{1}(t)=f_{1}(t)+n(t)
$$

The reference signal with the same frequency as the measured signal is defined as

$$
\mathrm{F}_{2}(t)=f_{2}(t)
$$

Where, $f_{1}(t)$ is the measured weak periodic signal, $n(t)$ is noise signal, $f_{2}(t)$ is the reference signal with the same frequency with $f_{1}(t)$, but $n(t)$ and $f_{2}(t)$ are irrelevant, the cross-correlation function $R_{F_{i} F_{2}}(\tau)$ is defined as

$$
\begin{aligned}
& R_{F_{1} F_{2}}(\tau)= \lim _{T \rightarrow \infty} \frac{1}{2 T} \int_{-T}^{T} F_{1}(t) F_{2}(t-\tau) d t \\
&=\lim _{T \rightarrow \infty}\left[\frac{1}{2 T} \int_{-T}^{T} f_{1}(t) f_{2}(t-\tau) d t\right. \\
&\left.\quad+\frac{1}{2 T} \int_{-T}^{T} n(t) f_{2}(t-\tau) d t\right] \\
&=R_{f_{1} f_{2}}(\tau)+R_{n f_{2}}(\tau)
\end{aligned}
$$

Where, $R_{f f f_{2}}(\tau)$ is the cross-correlation function of $f_{1}(t)$ and $f_{2}(t), R_{n f_{2}}(\tau)$ is the crosscorrelation function of $n(t)$ and $f_{2}(t)$, the reference signal $f_{2}(t)$ and noise signal $n(t)$ is uncorrelated in the ideal state, if the mean value of $n(t)$ or $f_{2}(t)$ is zero. The crosscorrelation function $R_{n f / 2}(\tau)$ can be considered as zero.

Assumption

$$
\begin{gathered}
\mathrm{F}_{1}(t)=f_{1}(t)+n(t)=A_{1} \sin \omega t+n(t) \\
\mathrm{F}_{2}(t)=f_{2}(t)=A_{2} \sin \omega t
\end{gathered}
$$

Where, $A_{1}$ is amplitude of the measured weak periodic signal $f_{1}(t), A_{2}$ is amplitude of the reference signal $f_{2}(t)$. Then we get

$$
R_{F_{1} F_{2}}(\tau)=R_{f_{1} f_{2}}(\tau)=\frac{A_{1} A_{2}}{2} \cos \omega \tau=A \cos \omega \tau
$$

Where, $A$ is the amplitude of cross-correlation function $R_{F_{1} F_{2}}(\tau)$. 
Thus, in the ideal state, the cross-correlation function calculation is the cosine signals with the same frequency as the measured periodic function. As noise hasn't any impact on periodicity of the signal, the cross-correlation detection method has a certain ability to filter noise signal. Through formula (8) the amplitude of the measured periodic signal can be obtained

$$
A_{1}=\frac{2 A}{A_{2}}
$$

But in fact, the noise signal cann't be completely uncorrelated with the reference signal, $R_{n f_{2}}(\tau)$ also may not is zero. Therefore, the cross-correlation function output is bound to contain some noise, which greatly limits the use of cross-correlation method to detect the noise ratio of weak signal.

\section{Weak Signal Detection Based On Compound System}

The weak signal detection method based on the relevant methods has the advantage of filtering part noise signal, the disadvantage is that the detection condition is the measured weak periodic signal with known frequency and the weak signal detected has the higher SNR threshold. The method of weak signal detection based on double coupled Duffing oscillator has the ability of the frequency detection of unknown measured periodic signal and extracting periodic signals submerged in the noise signal, disadvantage is that the strength of the noise have relatively strong influence on the system phase track observation. Therefore, this paper presents a composite system combining the two techniques for weak signal detection with unknown frequency that can extract the measured periodic signal from the strong noise better and can greatly reduce the SNR threshold of the measured signal.

Weak signal detection based on compound system divided into two links: frequency detection link and amplitude detection link, it is just like Fig.2. In the frequency detection, firstly the frequency of the measured periodic signal is detected through segmentation frequency detection method based on double coupled Duffing oscillator, providing the reference signal frequency for amplitude detection. Then measured signal including noise and given reference signal with the same frequency are made cross-correlation processing, filtering out part of the noise in the literature [12]. In the process of amplitude detection, firstly the above cross-correlation signal and the reference signal are treated again with the cross correlation processing, in order to get rid of noises. Finally, amplitude of double coupling Duffing systems is input into the output signal[13-16].

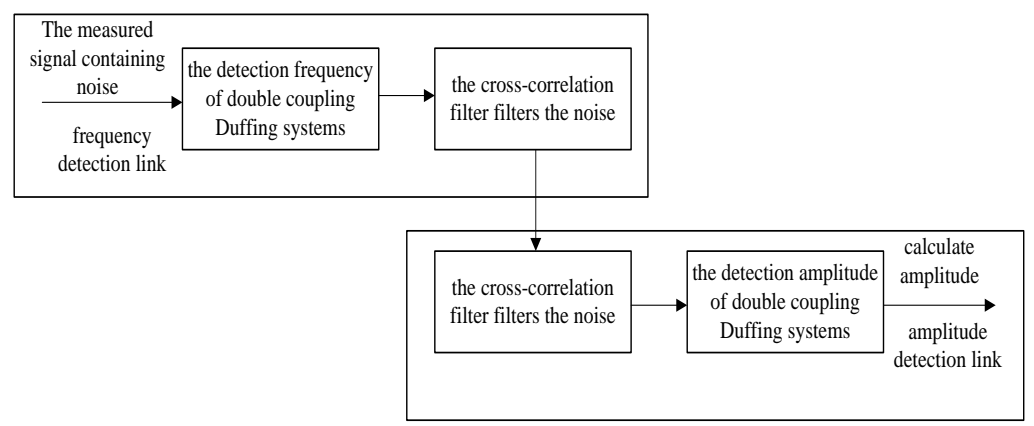

\section{Figure 2. The Basic Idea of the Weak Signal Detection Based on the Compound System}

The input measured signal mixed with noise is defined as

$$
\mathrm{F}_{1}(t)=f_{1}(t)+n(t)
$$


Where, $f_{1}(t)$ is the measured weak periodic signal with frequency $\omega$ and amplitude $A_{1} \cdot n(t)$ is noise signal.

Double coupling Duffing system model is defined as

$$
\left\{\begin{aligned}
\ddot{x}(t) & +k \dot{x}(t)-x(t)+x^{3}(t)+d(y(t)-x(t)) \\
& =F_{2}(t) \\
\ddot{y}(t) & +k \dot{y}(t)-y(t)+y^{3}(t)+d(x(t)-y(t)) \\
& =F_{2}(t)+F_{1}^{\prime}(t)
\end{aligned}\right.
$$

Where, $F_{2}(t)$ is the reference signal of subsection frequency detection method. The frequency $\omega$ of measured periodic signal is accurately measured in use of subsection frequency detection method based on double coupling Duffing oscillator proposed in the third chapter.

Reference signal $F_{3}(t)=f_{3}(t)$ has the frequency $\omega$ and amplitude $A_{3}$, primary cross-correlation function is defined as

$$
\begin{aligned}
R_{F_{1} F_{3}}(\tau)= & \lim _{T \rightarrow \infty} \frac{1}{2 T} \int_{-T}^{T} F_{1}(t) F_{3}(t-\tau) d t \\
= & \lim _{T \rightarrow \infty}\left[\frac{1}{2 T} \int_{-T}^{T} f_{1}(t) f_{3}(t-\tau) d t\right. \\
& \left.\quad+\frac{1}{2 T} \int_{-T}^{T} n(t) f_{3}(t-\tau) d t\right] \\
= & R_{f_{1} f_{3}}(\tau)+R_{n f_{3}}(\tau)
\end{aligned}
$$

Where, the $R_{f_{1} f_{3}}(\tau)$ can be regarded as part of periodic signal, $R_{n f_{3}}(\tau)$ can be regarded as residual of noise signal. Therefore, the equation (13) is written as

$$
\mathrm{F}_{1}^{\prime}(t)=f_{1}^{\prime}(t)+n^{\prime}(t)
$$

Where, $\mathrm{F}_{1}^{\prime}(t)$ is the primary signal processing measured primary cross-correlation processing with frequency $\omega$ and amplitude $A_{2}, f_{1}^{\prime}(t)$ is periodic signal, $n^{\prime}(t)$ is the residual of noise signal.

$$
\begin{gathered}
f_{1}^{\prime}(t)=R_{f_{1} f_{3}}(\tau) \\
n^{\prime}(t)=R_{n f_{3}}(\tau) \\
A_{2}=\frac{A_{1} A_{3}}{2}
\end{gathered}
$$

The reference signal $F_{3}(t)=f_{3}(t)$ has frequency $\omega$ and amplitude $A_{3}$, the secondary cross-correlation function is defined as

$$
\begin{aligned}
& R_{F_{1}^{\prime} F_{3}}(\tau)= \lim _{T \rightarrow \infty} \frac{1}{2 T} \int_{-T}^{T} F_{1}^{\prime}(t) F_{3}(t-\tau) d t \\
&=\lim _{T \rightarrow \infty}\left[\frac{1}{2 T} \int_{-T}^{T} f_{1}^{\prime}(t) f_{3}(t-\tau) d t\right. \\
&\left.\quad+\frac{1}{2 T} \int_{-T}^{T} n^{\prime}(t) f_{3}(t-\tau) d t\right] \\
&=R_{f_{1}^{\prime} f_{3}}(\tau)+R_{n^{\prime} f_{3}}(\tau)
\end{aligned}
$$

Where, $R_{f_{1}^{\prime} f_{3}}(\tau)$ is part of periodic signal, $R_{n^{\prime} s_{3}}(\tau)$ is the residual of noise signal. Therefore, the equation (18) is written as

$$
\mathrm{F}_{1}^{\prime \prime}(t)=f_{1}^{\prime \prime}(t)+n^{\prime \prime}(t)
$$

Where, $\mathrm{F}_{1}^{\prime \prime}(t)$ is the secondary processing signal measured the secondary crosscorrelation processing with frequency $\omega$ and amplitude $A_{4} \cdot f_{1}^{\prime \prime}(t)$ is periodic signal, $n^{\prime \prime}(t)$ is the residual of noise signal. 


$$
\begin{gathered}
f_{1}^{\prime \prime}(t)=R_{f_{1}^{\prime} f_{3}}(\tau) \\
n^{\prime \prime}(t)=R_{n^{\prime} f_{3}}(\tau) \\
A_{4}=\frac{A_{2} A_{3}}{2}=\frac{\frac{A_{1} A_{3}}{2} A_{3}}{2}=\frac{A_{1} A_{3}^{2}}{4}
\end{gathered}
$$

The $F_{1}^{\prime \prime}(t)$ is input to double coupling Duffing system, then we get

$$
\left\{\begin{aligned}
\ddot{x}(t) & +k \dot{x}(t)-x(t)+x^{3}(t)+d(y(t)-x(t)) \\
& =F_{2}^{\prime}(t) \\
\ddot{y}(t) & +k \dot{y}(t)-y(t)+y^{3}(t)+d(x(t)-y(t)) \\
& =F_{2}^{\prime}(t)+F_{1}^{\prime \prime}(t)
\end{aligned}\right.
$$

Where, $F_{2}^{\prime}(t)$ is the reference signal with frequency $\omega$ and the critical amplitude $A_{d}, \mathrm{~F}_{1}^{\prime \prime}(t)$ is the measured signal with amplitude $A_{4}$. Finally, amplitude of the original measured periodic signals can be got according to the formula (22).

$$
A_{1}=\frac{4 A_{4}}{A_{3}^{2}}
$$

Based on the above calculations, we can get the following weak signal detection method based on complex system theory as follows.

Theorem one: if the measured weak periodic signal with frequency $\omega$ and amplitude $A_{1}$ is input into the composite system, composed of the primary processing signal with the same frequency, but with amplitude $A_{3}$, and the secondary processing signal with the same frequency, but with amplitude $A_{4}$. The amplitude of the measured signal can be measured as

$$
A_{1}=\frac{4 A_{4}}{A_{3}^{2}}
$$

\section{Simulation Analysis}

The simulation experiment makes use of weak signal detection method based on compound system, it does simulation analysis with input of amplitude 0.05 and frequency $1.5 \mathrm{rad} / \mathrm{s}$ (The unit of frequency covered in this chapter is rad/s) sinusoidal signal and strength 0.001 (the amplitude is about 0.3) white gaussion noise signal. The measured signal as the input is

$$
f_{1}(t)=0.05 \sin 1.5 t
$$

The input signal without noise is shown in Figure 3 (a), the input signal with noise is shown in Fig 3 (b).

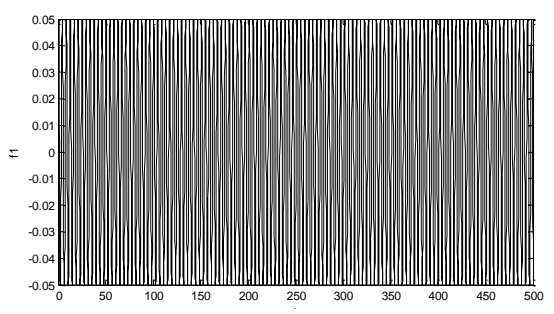

Figure 3. (a) The Input Signal without Noise 


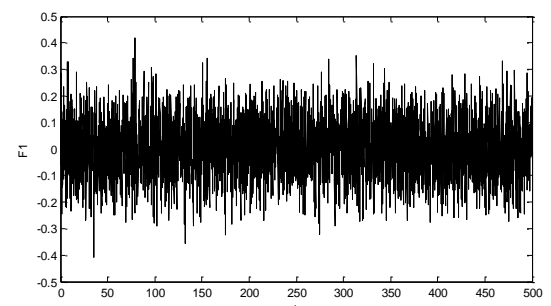

Figure 3. (b) The Input Signal with Noise

a) The measured signal is input to the segmented detection frequency measurement system.

Simulation results show that the intermittent chaotic phenomenon occurs between 1.4685 and 1.5126, it is shown in Figure 4

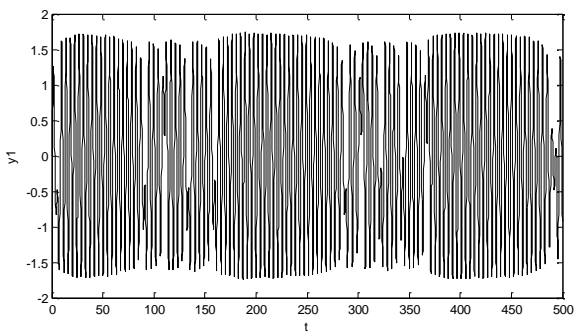

Figure 4. (a) The Intermittent Chaos Phenomenon when the Reference Frequency is 1.4685

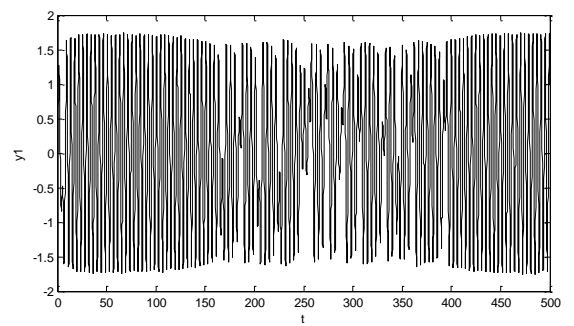

Figure 4. (b) The Intermittent Chaos Phenomenon when the Reference Frequency is 2.5751

It is measured that

(1) When reference frequency is $\omega_{k}=1.4685 \mathrm{rad} / \mathrm{s}$ intermittent chaos periodic is $T_{\Delta 1}=213 s, \Delta \omega_{1}=2 \pi / T_{\Delta 1}=0.0295$

(2) When the reference frequency is $\omega_{k+1}=1.5126 \mathrm{rad} / \mathrm{s}$, intermittent chaos periodic is $T_{\Delta 2}=397 \mathrm{~s}, \Delta \omega_{2}=2 \pi / T_{\Delta 2}=0.0158$

The measured signal frequency is calculated as

$$
\begin{aligned}
\omega & =\frac{\left(\omega_{k}+\Delta \omega_{1}\right)+\left(\omega_{k+1}-\Delta \omega_{2}\right)}{2} \\
& =\frac{(1.4685+0.0295)+(1.5126-0.0158)}{2} \\
& =1.4974 \mathrm{rad} / \mathrm{s}
\end{aligned}
$$

Therefore, the measured signal frequency is $\omega=1.4974 \mathrm{rad} / \mathrm{s}$.

b) The reference signal of cross-correlation function is set of the $1.4974 \mathrm{rad} / \mathrm{s}$ frequency and 2 amplitude, the signal which is the measured signal treated with two levels cross-correlation processing through cross-correlation function is shown in Fig.5 


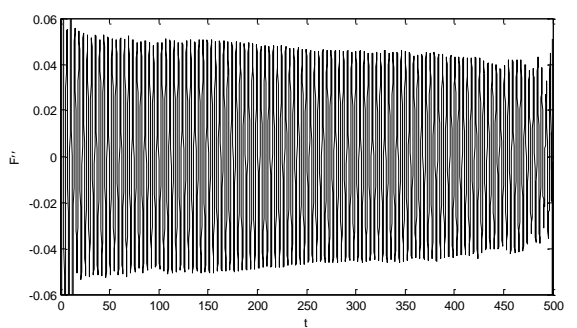

Figure 5. The Signal of the Second Level

c) When the reference signal $F_{2}^{\prime}(t)$ frequency is $1.4974 \mathrm{rad} / \mathrm{s}$ and the amplitude is set as the critical value of 0.82673 , the system trajectory is at the critical chaos state. The secondary processing signal is input to double coupling Duffing system, the system trajectory change into the large-scale periodic state, it is shown in Fig.6. the system trajectories is back at chaotic state and large-scale periodic state as shown in Fig.7 through modulating amplitude of reference signal, we can get the amplitude $A_{5}=0.78051$ of reference signal. Amplitude of the secondary processing signal is calculated as

$$
A_{4}=A_{d}-A_{5}=0.82673-0.78051=0.04622
$$

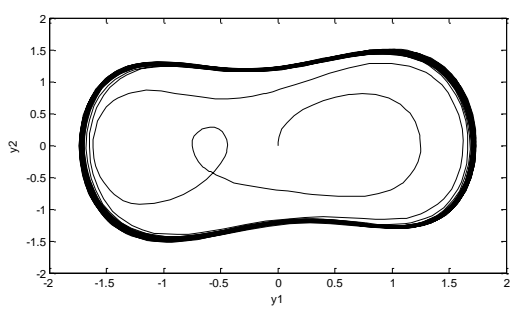

Figure 6. The System Phase Track after the Second Level Signal Input

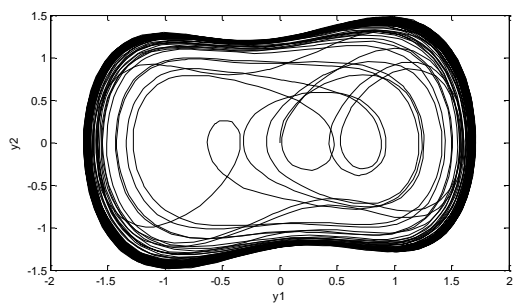

Figure 7. The System Phase Track after Adjusting the Reference Signal Amplitude

d) The amplitude of the original measured periodic signals is

$$
A_{1}=\frac{4 A_{4}}{A_{3}^{2}}=\frac{4 * 0.04622}{2 * 2}=0.04622
$$

Finally we can measure the frequency $1.4974 \mathrm{rad} / \mathrm{s}$ and amplitude 0.04622 of the measured periodic signal, frequency and amplitude measured are accurate compared with the actual input signal with frequency $1.5 \mathrm{rad} / \mathrm{s}$ and amplitude 0.05 .

\section{Conclusion}

Through studying the advantage and disadvantage of weak signal detection method based on double coupling Duffing oscillator and cross-correlation detection method, this paper proposes a compound system for weak signal detection with unknown frequency. The compound system is combined with correlation method 
and double coupling Duffing oscillator, correlation method has the advantage of filtering part of noise signal, double coupling Duffing oscillator has the advantage of testing the measured periodic signal with unknown frequency and extracting the periodic signal submerged in noise signal. Through a series of simulation experiments, it shows that the system can better extract the measured periodic signal from strong noise and can greatly reduce the SNR threshold of the signal. This method not only solves the problem that weak signal detection method based on chaotic system easily generates the misjudgment affected by noise, but also breaks through the limitations that the existing chaotic oscillator detection method can only detect signal with known frequency.

\section{References}

[1] S. Peiming, D. Xuejuan and H. Dongying, "Study on multi-frequency weak signal detection method based on stochastic resonance tuning by multi-scale noise [J]", MEASUREMENT, vol. 47, (2014), pp. 540-546.

[2] S. Bin, R. ChaoHuang and W. Chao, "Stochastic resonance in multi-frequency weak signal detection with alpha stable noise [J]", ACTA PHYSICA SINICA, vol. 62, no. 21, (2013).

[3] S.-H. Shi, Y. Yong and H. Wang, "Weak Signal Frequency Detection Method Based on Generalized Duffing Oscillato [J]", Chinese Physics Letters, vol. 28, no. 4, (2011).

[4] C. S. Le and S. Fabien, "s markov tree frequency selectivity for the detection of weak transient signals[J]", Traitement du signal, vol. 4, no. 5, (2010), pp. 443-482.

[5] D. S. Yang, L. Li and J. Yang, "the weak signal detection with unknown frequency based on double coupling chaotic oscillator", Journal of Northeastern University: Natural Science Edition, (2012).

[6] K. L. Zhang and H. M. Zhu, "Weak signal detection technology [J]", Avionics Technology, vol. 40, no. 2, (2009), pp. 30-36

[7] Z. Rongbiao, H. Haiyan and F. Youbing, "Study on weak signal detection method based on wavelet entropy [J]", Chinese Journal of Scientific Instrument, vol. 28, no. 11, (2007), 2078.

[8] M. Ogorzalek, "Taming chaos. I. Synchronization [J]", IEEE Trans. Circuits Syst. I, Fundam. Theory Appl., vol. 40, no. 10, (1993), pp. 693-699.

[9] Z. Zhu, H. Leung and Z. Ding, "Optimal synchronization of chaotic systems in noise [J]", IEEE Trans. Circuits Syst. I Fundam. Theory Appl., vol. 46, no. 11, (1999), pp. 1320-1329.

[10] L. M. Pecora and T. L. Carroll, "Synchronization in chaotic systems [J]", Phys. Rev. E, Stat. Phys. Plasmas Fluids Relat. Interdiscip. Top., vol. 64, no. 8, (1990), pp. 821-824.

[11] T. Li"synchronization and its application in secure communication [J]", Journal of Hubei Radio and TV University, vol. 31, no. 4, (2011), pp. 158-159.

[12] Q. F. Shang, C. Q. Yi and S. L. Li, "Study on detection of weak sinusoidal signal based on Duffing oscillator [J]", Chinese Journal of Electrical Engineering, vol. 25, no. 2, (2005), pp. 66-70.

[13] W L. Zhao, Y P. Yin and Q. Fang, "Weak signal detection based on stochastic resonance of piecewise model [C]", Advanced Engineering Forum, vol. 4, (2012), pp. 222-226.

[14] R. Li and W. Min, "Research on weak signal detection method under the strong noise background [J]", Information Technology, vol. 2, no. 17, (2012).

[15] N. C. Yan and S. Y. Wu, "Study on weak signal detection based on correlation detection and chaos theory [J]", Chinese Journal of scientific instrument, vol. 22, no. 1, (2001), pp. 32-35

[16] J. L. Man and H. J. Zhang, "Research and Realization of signal detection based on correlation [J]", Journal of Jilin Teachers Institute of engineering and technology, vol. 6, no. 011, (2004).

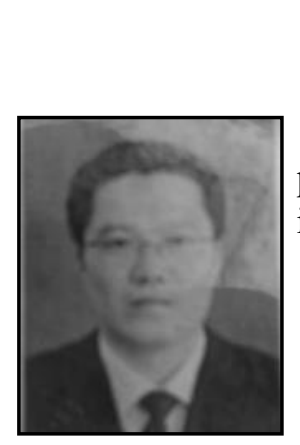

\section{Authors}

Hongyu Zhang, He comes from state grid Shenyang electric power supply company, Shenyang, China. His research interests include power system control, control theory and control engineering. 


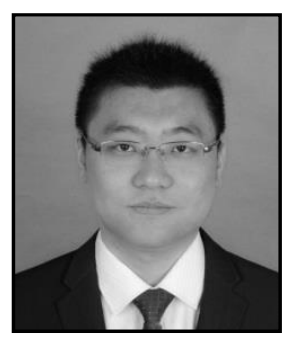

Yihe Wang, He comes from state grid Shenyang electric power supply company, Shenyang, China. His research interests include power system control, grid control and automation of electric power systems.

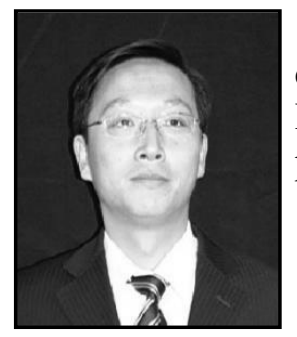

Xingyu Liu, He is the director of sales department's marketing department at state grid Liaoning power company, Shenyang, China. His research interests include scheduling of electrical power, smart power grid, planning, energy management and grey theory.

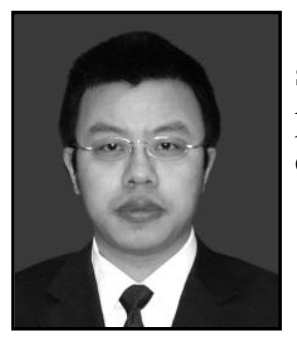

Gang Zhao, He comes from state grid Shenyang electric power supply company, Shenyang, China. His research interests include power system fault detection, control engineering and energy optimization.

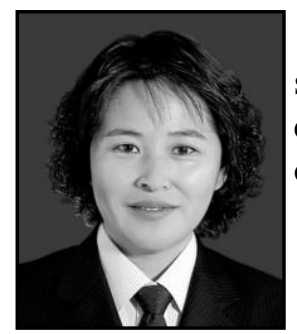

Meiyan Shan, She comes from state grid Shenyang electric power supply company, Shenyang, China. Her research interests include compensation of reactive power, power dispatching and energy optimization. 